\title{
Gas-Liquid-Solid Phase Transition Model for Two-Dimensional Nanocrystal Self-Assembly on Graphite
}

\author{
Jing Tang, Guanglu Ge, and Louis E. Brus* \\ Department of Chemistry, Columbia University, New York, New York 10027
}

Received: December 18, 2001; In Final Form: March 29, 2002

\begin{abstract}
The self-assembly of $12 \mathrm{~nm}$ PbSe nanocrystals on a graphite surface during solvent evaporation is analyzed within the two-dimensional van der Waals phase condensation model. The interparticle attractive force, phase separation kinetics, and critical coverage in this system can be revealed from aggregation spatial patterns at different kinetic stages. Cracking in nanocrystal monolayer drying at high coverage reveals limitations of this model.
\end{abstract}

\section{Introduction}

The assembly of nanocrystals from solution into ordered arrays is a promising approach toward advanced materials, ${ }^{1}$ and an important fundamental problem by itself, in which various interactions between nanocrystals, substrates, and solvents are involved. General aspects of the forces between nanocrystals can be obtained from aggregate morphologies and diffraction data. $^{2-5}$ Ohara et al. showed that size-dependent van der Waals (vdW) attraction between dodecanethiol-coated Ag nanoparticles is sufficient to induce size-segregation and reversible superlattice formation. ${ }^{2}$ Kogel et al. used a soft-sphere model combining vdW attraction and steric repulsion to fit observed structure function from SAXS measurements. ${ }^{4}$ Theoretical calculations based on ab initio methods also exist and give insights in these interactions. $^{6-7}$

However, often the organization process is dominated by the nanocrystal/substrate interaction, by drying kinetics, and/or by the solution/substrate interfacial energy. Substrate roughness, solvent dewetting, ${ }^{8}$ and convective flow ${ }^{9}$ can create unusual nonequilibrium structures such as mesoscopic rings and honeycomb nanocrystal networks. Information about the phase diagram and pair interaction potential of solvated nanocrystals cannot be easily obtained from these studies. In contrast to these systems, the organization of $4 \mathrm{~nm}$ alkane-capped CdSe nanocrystals on graphite surfaces can be understood as an equilibrium phase separation, dominated by the nanocrystal-nanocrystal interaction. ${ }^{10}$ Here the nanocrystal solution completely wets the substrate. We have modeled this process as a fluid-fluid phase separation in a 2D Lennard Jones 6-12 particle system, governed by the universal phase diagram in Figure 1. Spinodal (barrierless) nucleation is observed when the system is cooled near the critical point $P_{\mathrm{c}}$, where the surface tension between the two fluid phases is very low. This phase transition model is "natural" in the limit of nanoparticle high surface mobility, without fixed bonds to the substrate. Graphite has very low surface roughness and friction. The nanocrystals are coated with a layer of alkane hair which lubricates the interface between the nanocrystal and the graphite; high mobility is observed even for dry nanocrystal aggregates on graphite. ${ }^{11}$ When the solvent is still present, this mobility should be even higher.

In this organization model, aggregation is a phase separation

*Corresponding author. E-mail: brus@chem.columbia.edu.

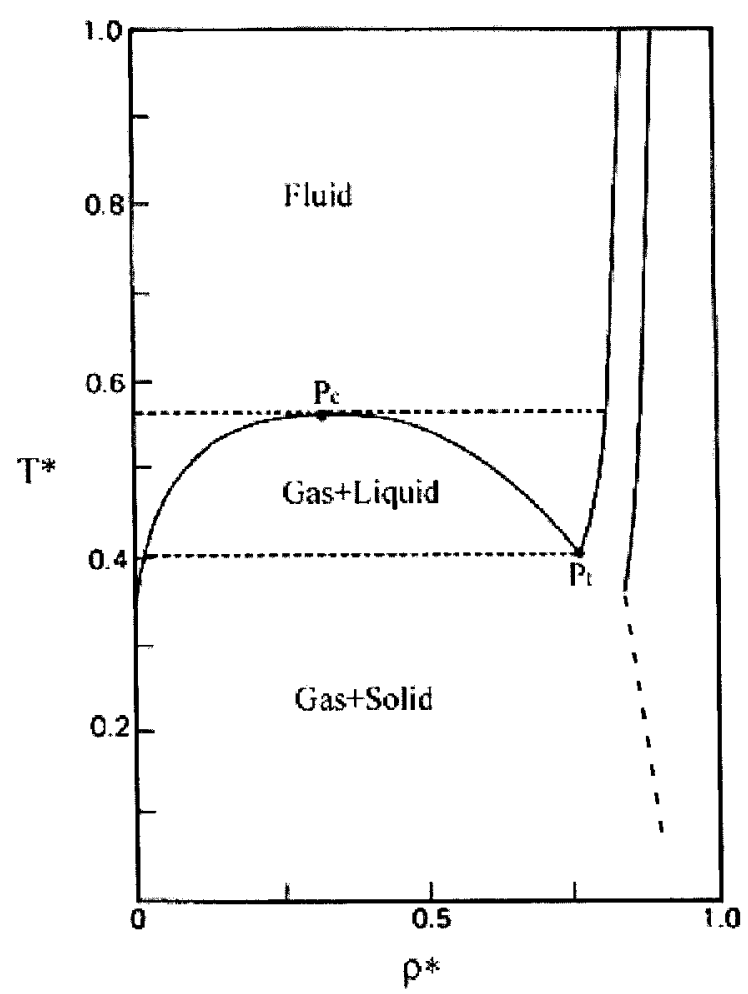

Figure 1. Universal phase diagram of 2-D L-J system adapted from ref $25 . P_{\mathrm{c}}$ is the critical point and $P_{\mathrm{t}}$ is the triple point. The vertical dotted line denotes the gas/solid coexistence curve extrapolated to $\rho^{*}$ 0.916 at $0 \mathrm{~K}$.

that is triggered by the increase of interaction between nanoparticles when a good solvent (hexane or chloroform) is replaced by a poor solvent (air). This interaction increase is functionally equivalent to a reduced temperature $T^{*}$ quench from a onephase (fluid) region into a two-phase (gas and liquid) region in Figure 1. Here $T^{*}=k T / \epsilon$ where $\epsilon$ is the nanocrystal pair bond energy. Under this framework, fundamental information about $\epsilon$ and the 2D nanocrystal phase diagram can be revealed by comparing experimentally observed patterns to those from computer simulations.

In this paper we extend our observations to far larger $12 \mathrm{~nm}$ cubic PbSe nanocrystals and find that this system also follows the phase separation mechanism. These PbSe nanocrystals are 

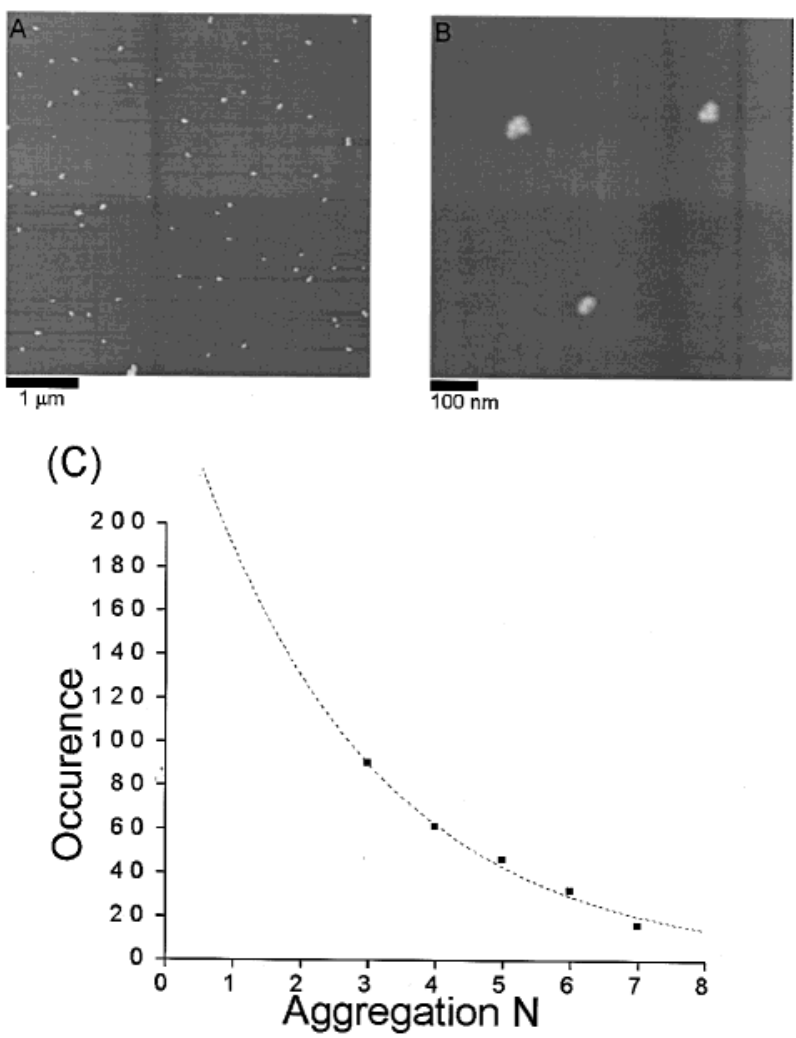

Figure 2. (A) Small aggregates of PbSe nanocrystals formed at surface coverage of $0.5 \%$ after drying of a hexane solution. (B) Example of a dimer, trimer, and quadramer identified from the high-resolution image. (C) Occurrence distribution of the aggregates formed by 3-7 nanoparticles. The distribution is fitted to the form $y=274( \pm 29)^{*} 0.69$ $( \pm 0.02)^{x}$ (dotted line).

big enough to be seen individually by AFM so that detailed information on the particle ordering in different patterns can be obtained, and the gaseous region (i.e., extremely low surface coverage) in the phase diagram can be explored. We also explore cracking that occurs when the liquid phase undergoes a liquid/ solid-phase transition (i.e., freezes) as $T^{*}$ drops below the triple point temperature $P_{\mathrm{t}}$. These observations help us to understand the limitations as well as the usefulness of the phase transition model.

\section{Experiment}

Twelve-nanometer cube-shaped PbSe nanocrystals capped with oleic acid were synthesized using an organometallic method by W. Gaschler and C. Murray of IBM Yorktown Heights. Sizeselection using the hexane/ethanol solvent pair was performed at least three times, both to narrow the size distribution (final standard deviation less than 5\%) and to reduce free oleic acid in solution. All solvents were of reagent grade (SAF) or better and used as received. Highly oriented pyrolytic graphite (HOPG) was used as the substrate. Tapping mode atomic force microscopy (AFM) images were taken using a Nanoscope III microscope (Digital Instruments, Santa Barbara, CA) after depositing a drop $(\sim 30 \mu \mathrm{L})$ of particle solution on HOPG and letting the drop dry in air. In the phase diagram, a complete nanocrystal monolayer at $0 \mathrm{~K}$ corresponds to unitless coverage $\rho^{*}=0.9165$, where $\rho^{*}$ is the area occupied by the particles per unit area. The AFM does not experimentally resolve interstitial spaces between nanocrystals in a complete monolayer, and thus a complete monolayer is $\rho^{*}=1$. We multiply our experimental coverages by 0.9165 to convert them to the theoretical $\rho^{*}$ scale.

\section{Results and Discussions}

TEM images show the PbSe nanocrystal cores are cubic in shape. However, in AFM images, the nanocrystals appear spherical. They also show hexagonal closest packing in arrays, as expected for spheres. We attribute this sphere-like behavior to the passivation of the nanocrystals by deformable oleic acid, which lowers the anisotropy of the interaction potential at the equilibrium bond length.

1. Extremely Dilute Regime: Evidence for Gas Phase. In the phase diagram only a single (gas) phase exists for a wide range of effective temperatures, if the surface coverage is very low, less than $1 \%$. The gas-phase equilibrium distribution of particles among monomers, dimers, or higher number aggregates (or among different geometries) reflects the interplay of enthalpy and entropy and can be used to measure the interparticle interaction. In our earlier study of $4 \mathrm{~nm} \mathrm{CdSe} \mathrm{nanocrystals,} \mathrm{we}$ did not observe small aggregates at low coverage, probably because they are easily swept away by the AFM tip during scanning. ${ }^{10}$ However, in Figure 2 we do observe small aggregates of the much more massive $12 \mathrm{~nm}$ PbSe particles. We plot the occurrence of the aggregates (aggregation number 3 to 7) as a function of their size, in large HOPG regions free of step edges. Monomers and dimers are omitted in this histogram because although they can be seen in pictures, they are still easy to be swept by the tip and preferentially bind at step edges.

This smoothly decaying distribution is expected in equilibrium self-assembly. For isotropic particles with bond energy $\epsilon=\alpha k T$, the equilibrium concentration (in mole fraction) of molecules in aggregates of aggregation number $N, X_{N}$ can be obtained based on: ${ }^{12}$

$$
\begin{aligned}
\mu=\mu_{1}^{\circ}+k T \log X_{1}=\mu_{2}^{\circ}+\frac{1}{2} k T \log \frac{1}{2} X_{2}= \\
\mu_{3}^{\circ}+\frac{1}{3} k T \log \frac{1}{3} X_{3}=\ldots
\end{aligned}
$$

where $\mu_{N}$ is the mean chemical potential of a nanocrystal in an aggregate of aggregation number $N$, with $\mu_{N}^{0}$ its standard part. $X_{N}$ is the volume fraction of aggregate of number $N$; in 2D it is the surface coverage. Also,

$$
\sum_{N=1}^{\infty} X_{N}=\text { total surface coverage of particle aggregates }
$$

We assume pairwise additive forces and that each extra particle forms two bonds when it joins an aggregate, thus lowering the free energy by $-2 \alpha k T$. For small $2 \mathrm{D}$ aggregates this assumption is valid. ${ }^{13}$ This gives

$$
X_{N}=N\left(X_{1}\right)^{N}\left(e^{\alpha}\right)^{2 N-3}
$$

The equivalent equation for a macroscopic model of large 2D aggregates is $X_{N}=N\left(X_{1} e^{\alpha}\right)^{N} e^{-\alpha \sqrt{N}} .12$ The aggregate concentration $C_{N}=X_{N} / N$ (not counting constitutional nanoparticles) forms a geometric series with progression of $X_{1} e^{2 \alpha} . X_{1}$ is extrapolated from the distribution curve and the total coverage. We find $\epsilon=$ $(3.5 \pm 0.1) k T$ from the fit in the figure. ${ }^{13}$

For comparison we calculate the van der Waals attraction between two bare $12 \mathrm{~nm}$ PbSe nanocrystal cores, separated by $4.5 \mathrm{~nm}$ of olecic acid as measured for close-packed arrays, from Lifshitz-Hamaker theory as described previously. ${ }^{2,4,10}$ The PbSe/ air/PbSe Hamaker constant is determined using literature optical constants,${ }^{14}$ and the imaginary part of PbSe's dielectric constant is modeled as a $\delta$ function centered at $2.5 \mathrm{eV}$. The calculated 

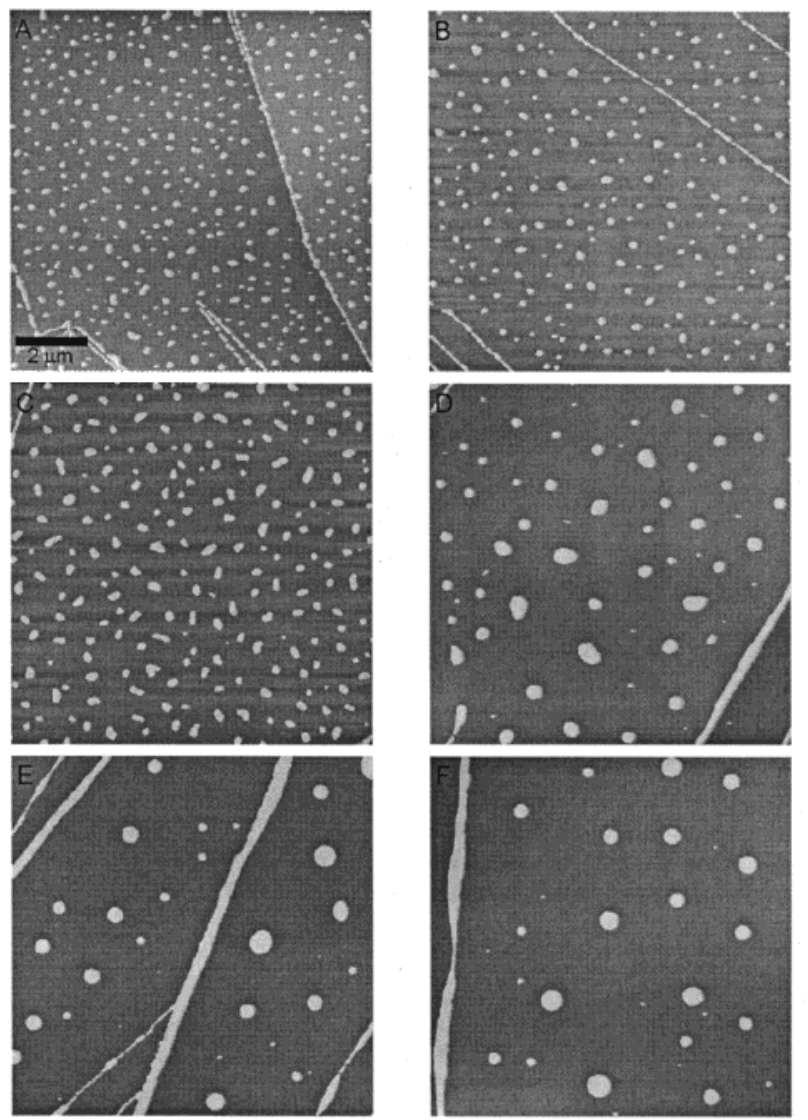

(G)

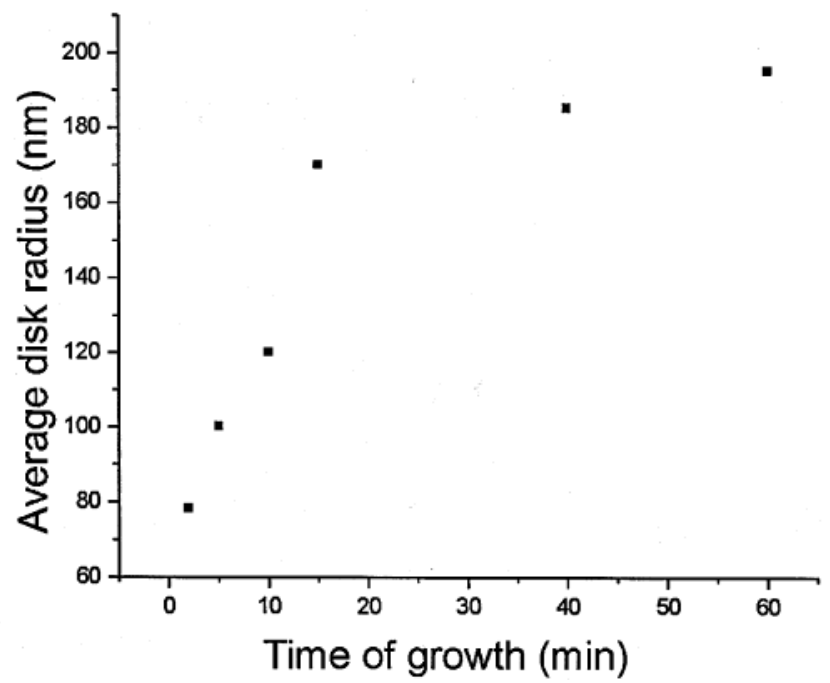

Figure 3. Figure $3 \mathrm{~A}-\mathrm{F}$ are representative images when the drying of an octane solution was quenched at certain times $t . t=2 \mathrm{~min}, 5 \mathrm{~min}$, $10 \mathrm{~min}, 15 \mathrm{~min}, 40 \mathrm{~min}$, and $60 \mathrm{~min}$ from $\mathrm{A}-\mathrm{F}$, respectively. All the images are of the same size. (B) Average disk size as a function of quenching time.

attraction energy is $1.6 \mathrm{kT}$. Our larger experimental value suggests that the dispersion force between two semiconductor cores is only a portion of the total energy. As argued by Leudtke and Landman, the interaction between capping molecules can be as large as the core van der Waals interaction after molecular conformations are optimized. ${ }^{9}$

In our phase separation model, organization into aggregates while drying corresponds to effective temperature cooling of the system. The smooth number distribution, together with the random spatial distribution, indicates that a thermal equilibrium is maintained in a large area as drying occurs. Further statistical studies could answer questions such as whether there are any magic numbers in forming these 2-D van der Waals clusters. The bond energy $\epsilon$ should increase during the drying process as the poor (air) solvent replaces the good (hexane) solvent. The bond energy $\epsilon=3.5 \mathrm{kT}$ and the distribution curve reflect the bonding in the final stage of drying, just before equilibrium is broken when the aggregates irreversibly bind to the surface and stop moving. These small aggregates could be useful to study coupling between nanocrystals as a function of aggregation number. For example, the surface diffusion constants of PbSe nanocrystal monomers, dimers and trimers have been measured and they decrease with the aggregate size. ${ }^{15}$

2. Nucleation, Growth, and Freezing at Intermediate Coverages. Twelve-nanometer PbSe nanocrystals show the same pattern-coverage dependence as $4 \mathrm{~nm}$ CdSe nanocrystals. We observe round disks at 5-20\% coverages, bicontinuous ribbon patterns at $20-40 \%$, and holely monolayers at larger coverages. Monolayer-thick round disks, composed of tens to hundreds of nanocrystals, appear as shown in Figure 4. The formation of disks results from homogeneous "liquid phase" nucleation and phase separation as the effective temperature cools. ${ }^{10}$ "Liquid phase" in Figure 1 is a condensed disordered nanocrystal/solvent phase of lower 2D nanocrystal density than the condensed ordered solid nanocrystal/solvent phase, which exists in the phase diagram at the same $T^{*}$ at higher coverage. Critical droplets form first and these droplets grow and coalescence toward the equilibrium of liquid-gas coexistence, until the disks irreversibly bind to the graphite in the last stages of drying.

Previous studies on the dynamics of nanocrystal selfassembly, using small-angle X-ray scattering and IR spectroscopy, ${ }^{16,17}$ focused on solvent evaporation and particle densification in a thick nanocrystal film at the late stage of drying. The initial nucleation and growth of 2D nanocrystal disks and the growth law have not been reported to the best of our knowledge. We performed a qualitative study using a simple stop-and-look method. Octane is used as the solvent and the drying is done in a closed container. Growth is stopped by opening the container and exposing the solution to air at certain times. As shown in Figure 3, the number of disks decreases drastically as growing time increases from $2 \mathrm{~min}$ to $40 \mathrm{~min}$, while the disks get larger and round at the same time. The surface coverage remains the same in these images. No obvious change is found when quenching time increases from $40 \mathrm{~min}$ to $60 \mathrm{~min}$ except that fewer small disks are left. These findings provide further evidence that nanocrystals assemble through the nucleation and coarsening as in a fluid-fluid phase separation. Computer studies of 2D Lennard-Jones particle systems show a similar evolution. ${ }^{18}$ In our earlier study of $4 \mathrm{~nm} \mathrm{CdSe}$ nanocrystals at similar coverages, we obtained images of liquid disks apparently caught in the act of fusing (Figure 2e of ref $10)$.

The shape and size of the disks differ with solvent. As shown in Figure 4, when octane was used, round disks form while much smaller disks of irregular, more faceted shape form when hexane or chloroform were used under the same coverage. This difference appears to reflect a longer drying time in octane, where the biggest observed disk had a diameter of $1.6 \mu \mathrm{m}$, than in chloroform or hexane. This argument is based on the different solvent evaporation rates, as the viscosity coefficients of these solvents are very close at room temperature. From the higher resolution AFM images of the disks, the nanocrystals show hexagonal close-packing after drying. The triple point $P_{\mathrm{t}}$ occurs 


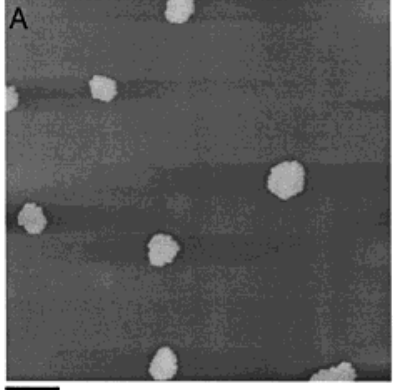

$200 \mathrm{~nm}$

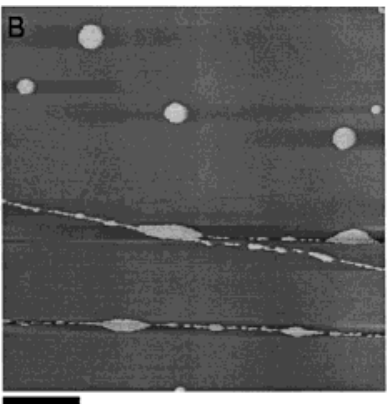

$1 \mu \mathrm{m}$

Figure 4. Disks formed in chloroform (A) and in octane (B). Disks formed in chloroform are smaller and faceted while in octane the disks are round. Coverages of both images are about $3 \%$.
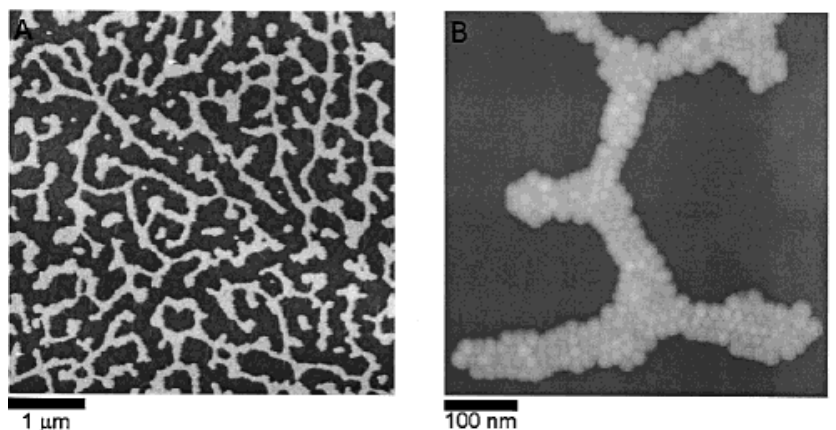

Figure 5. Representatvie spinodal structure (coverage is 35\% and solvent used is hexane). (B) A high-resolution image showing individual particles in the ribbon structure.

at about $T^{*}=0.41$ and $\rho^{*}=0.78$. In section I we found $\epsilon=$ $3.5 \mathrm{kT}$, at the end of the drying process when particles stop moving. This means that as drying finishes, the system cools below its triple point; liquid droplets formed during drying should freeze. Finite-size thermodynamic effects involving the surface energy between gas and liquid should depress the freezing temperature in small droplets. Nevertheless, we attribute the internal polycrystalline nature of the disks (and spinodal ribbons in the following section) to freezing of liquid drops formed at higher $T^{*}$. If particles are dried from an octane solution, the system can stay in the liquid $T^{*}$ region longer and large disks grow. When these freeze, the round liquid morphology remains. Previous work has shown that very large-sized internally crystalline monolayers are obtained in drying of highboiling-point solvents. ${ }^{19}$ In hexane or chloroform, small nanocrystal droplets freeze and develop facets, as surface particles need move only a short distance. Even in octane, smaller disks at early stages are more faceted as shown in Figure 3A-C.

Nanocrystals occupying the second layer are rarely found. As argued by Korgel and Fitzmaurice, ${ }^{3}$ this implies a strong wetting of PbSe nanocrystal solutions on graphite. Dewetting patterns (i.e., rings of nanocrystals) are never observed on graphite. One possible reason is that nanoparticles have high mobility and dewetting holes cannot get pinned and manifest themselves. ${ }^{2}$ Also the organic solvents strongly wet the thick graphite substrate.

When the solvent is still present, nanocrystals experience a weaker vdW attraction due to the dielectric shielding of solvent molecules. ${ }^{10}$ This corresponds to a higher effective temperature $T^{*}(=k T / \epsilon)$. As in the earlier CdSe work, we carried out imaging under organic solvents using the Bioscope liquid cell. Small aggregates of $\mathrm{PbSe}$ nanocrystals are observed together with single particles as shown in Figure 7. Aggregates do not grow with time. This is consistent with a weaker attraction between

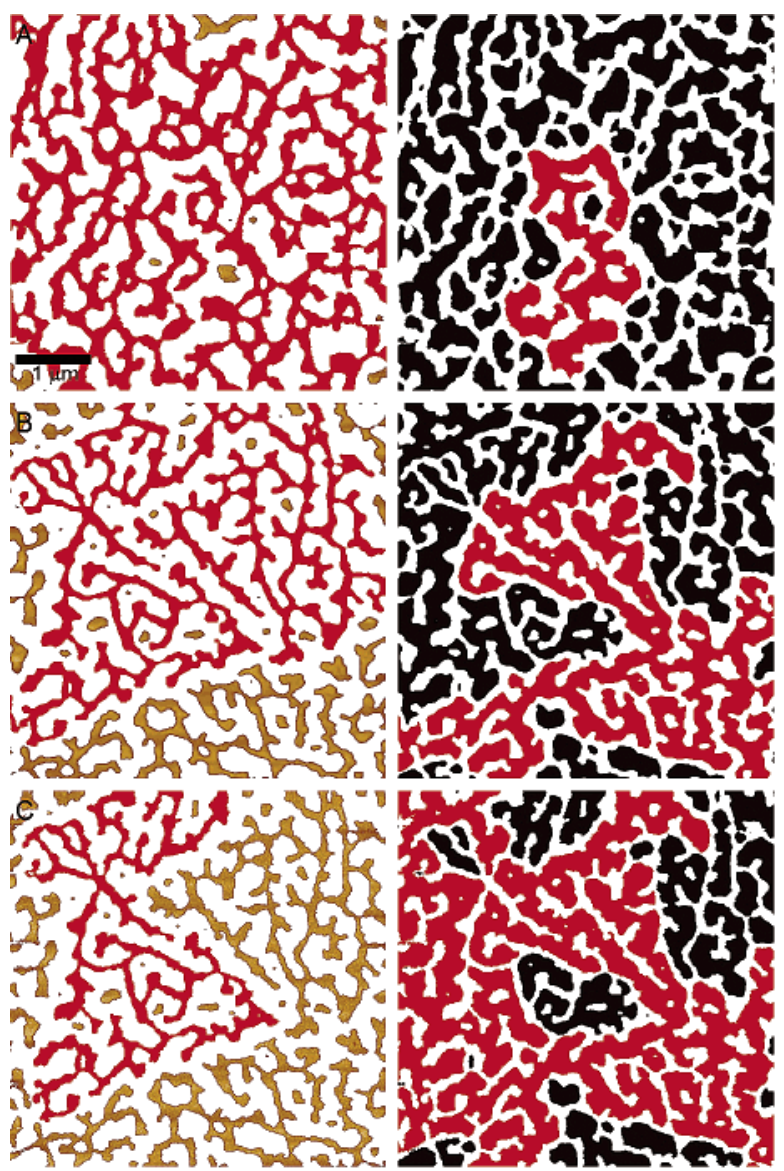

Figure 6. Largest connected clusters (red color in both panels, the left one represents the particles while the right one represents the substrate) as a function of surface coverage. Surface coverages in $(\mathrm{A}-\mathrm{C})$ are $39 \%, 35 \%$, and $32 \%$, respectively.

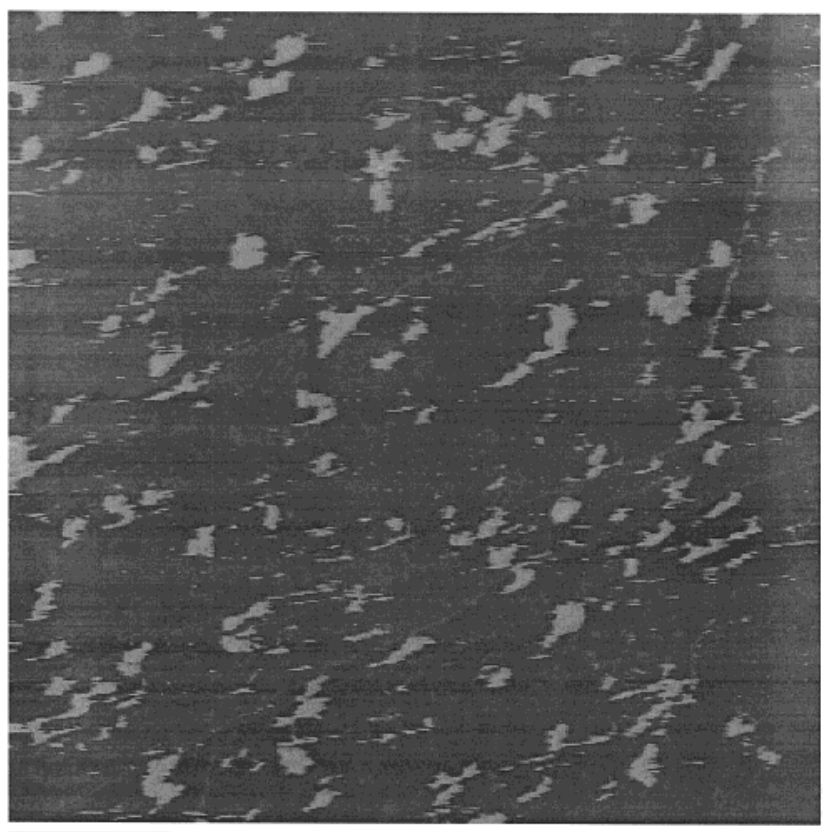

$1 \mu \mathrm{m}$

Figure 7. PbSe nanocrystals form small aggregates under liquid. In situ imaging is performed on a Bioscope AFM in an open configuration.

nanocrystals. In principle, the number distribution analysis can be performed to determine the exact interparticle attractive force, as in section 1 , but the poor quality of images hinders an 

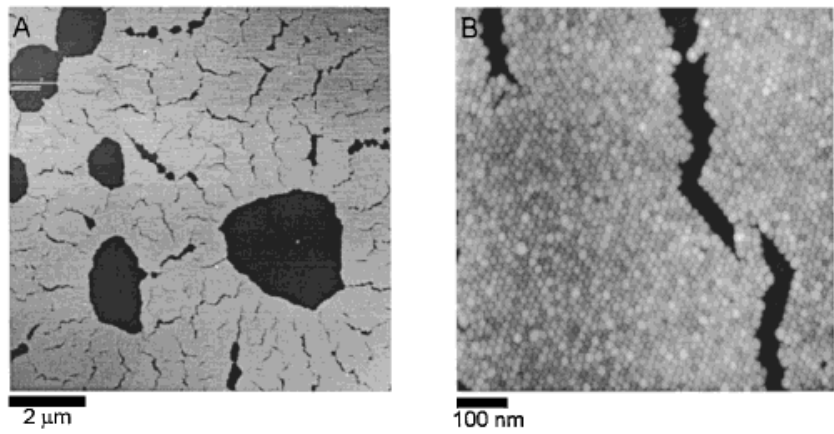

Figure 8. (A) AFM image of cracks formed in the monolayer film of $\mathrm{PbSe}$ nanocrystals after drying (coverage of $75 \%$ ). (B) High-resolution image of the cracks with individual nanocrystals clearly seen.

accurate count of aggregate sizes. We suspect the tip disturbance on the particles is bigger in solution than on dry samples.

3. Connectivity from Spinodal Patterns. Regular bicontinuous patterns which resemble polymer spinodal decomposition patterns were observed at $20-40 \%$ coverage. Representative images are shown in Figure 5A. The high-resolution image in Figure 5B allows us to determine the accurate orientations of individual nanoparticles within the aggregates.

Similar bicontinuous images are observed in computer simulations at early times when the particle systems cool into the gas-liquid region close to $P_{\mathrm{c}}{ }^{18} \mathrm{~A}$ simple connectivity analysis was carried out to determine $P_{\mathrm{c}}$, where both the particle phase and bare substrate (particle gaseous phase) should form connected networks across the whole area. We labeled the largest clusters in both phases as shown in Figure 6. When the coverage decreases from $41 \%$ to $31 \%$, the connectivity of particle domains decreases whereas that of substrates increases. Note that the shape of these clusters remains fractal. It is at around $34 \%$ that both reach large connection. This value is close to the theoretical prediction of $32.5 \%$ for a $6-12 \mathrm{~L}-\mathrm{J}$ particle system. However the inaccuracies in measuring surface coverage due to tip convolution need to be considered. A similar connectivity method was used to determine percolation threshold in a metal/insulator film..$^{20}$ Critical parameters are usually hard to investigate because of extreme conditions and fast evolution.

As the coverage continues to increase above $45 \%$, spinodal morphologies are not seen. Instead, round holes appear inside a continuous layer of nanoparticles. This has been previously observed in CdSe particle systems. In computer simulations this morphology represents gas bubbles inside a liquid phase during the drying process. From repeated AFM measurement, the distance between the adjacent nanoparticles is $16.8 \pm 0.2 \mathrm{~nm}$. The thickness of one oleic acid layer is around $2 \mathrm{~nm}$. This means that the nanocrystals are closed packed and the shape of domain is harder to anneal compared to inside.

4. Cracking in Large Area Monolayers. Cracking in drying of thin solid films is due to the contract of the material and the consequent stress in the film. It reveals information of the bonding between components and the interaction between the film and underneath substrate. ${ }^{21}$ In our system, cracks are always observed when a monolayer of nanocrystals dries at coverages above about $64 \%$ as shown in Figure 8 . The appearance of cracking directly shows that the bond length between nanocrystals is shorter, and the nanocrystal density $\rho^{*}$ larger, when organic solvent is replaced by air. Cracking also indicates that nanocrystals do bond to the surface in the last stages of drying, because freely contracting films do not crack. Such bonding is not considered in the gas-liquid-solid phase transition model we describe. Bonding to the surface will hinder the nanocrystal random thermal relative motion that characterizes a true liquid phase, and also stop disk (or spinodal stripe) mobility and coarsening. In the phase diagram, the liquid/solid freezing is a first-order phase transition; bonding to the surface may complicate this process. Computer simulations of 2D melting show the transition is complex, and sensitive to the exact form of the pair potential, and to the number of particles in the simulation. ${ }^{22-25}$ Finite size thermodynamic effects appear to be important.

Freezing processes can occur to both the right and left of $\mathrm{P}_{\mathrm{t}}$ in Figure 1. As $T^{*}$ drops to the right of $P_{\mathrm{t}}$, the system first crosses the liquid/solid coexistence line. Solid-phase crystallites should precipitate out of a liquid phase. Condensed phase liquid and solid coexist over a range of $T^{*}$ until the triple point temperature is reached. At this point the remaining liquid freezes, creating bubbles of very low-density gas phase. We observed cracking in this coverage range.

To the left of $P_{\mathrm{t}}$, the phase diagram predicts that the system is in gas/liquid equilibrium above the triple point temperature $T_{\mathrm{t}}$. The entirely liquid monolayer freezes suddenly upon cooling below $T_{\mathrm{t}}$. In fact cracks are not observed when a large area dries in the coverage range $45-64 \%$. We might attribute this difference in cracking behavior as related to the expected difference in solid nucleation process above and below the triple point coverage. Further experimental work is needed to explore this hypothesis, and to understand the effect of finite size thermodynamic corrections, and of nanocrystal bonding to the surface. We do not know how closely the nanocrystal potential with ligands corresponds to the Lennard-Jones 6-12 potential; at high coverages the exact potential shape will affect the liquid-solid phase diagram.

The number density of cracks observed is uniform over wide areas, which implies that solvent molecules evaporate uniformly from the surface of the film. In standard theory, the direction of cracks is perpendicular to the local stress vector, which is determined by the drying front. ${ }^{26}$ In large free area cracks point randomly but point to big gaps (e.g., round holes) if they are nearby. The direction of stress is perpendicular to open edges. ${ }^{26}$ As to the shape of cracks, they are rarely straight lines. Rather they are wavy and have different length and width. The dynamics of crack formation is not revealed in our experiment. But comparison to drying of a monolayer of micron-sized polysterene spheres ${ }^{27}$ suggests these cracks appear sequentially. Sparse and straight cracks are symbolic of early nucleation stage. On the other hand, a late stage of cracking after complete development should show a ladder pattern of cracks: cracks divide the layer into uniform cells. Clearly the cracking in our nanocrystal system is stopped in an intermediate stage. There appears to be a scaling relation between the length and width of the cracks.

The average spacing between the cracks in the final dry film, as shown in Figure 9, appears to be uniform across the film, and increases in the two layer region than the monolayer region. This has been previously observed in drying of a mud layer where the spacing between cracks is proportional to the layer thickness. ${ }^{28}$ For the first layer, the spacing is around $200 \mathrm{~nm}$.

Crack patterns are determined by the stress pattern in the film. Often a discrete spring-block model is used to simulate an elastic layer. ${ }^{27}$ In this model particles/nodes bind to each other through spring 1 with spring constant $k_{1}$, and they attach to surface elements through spring 2 (with a smaller spring constant, $k_{2}$ ). Cracks open when the strain energy is larger than the bonding energy between two particles (spring 1 breaks). In ref 21, the sliding along the surface is also considered. Before breakage particles could slide on the substrate. In a one-dimensional 

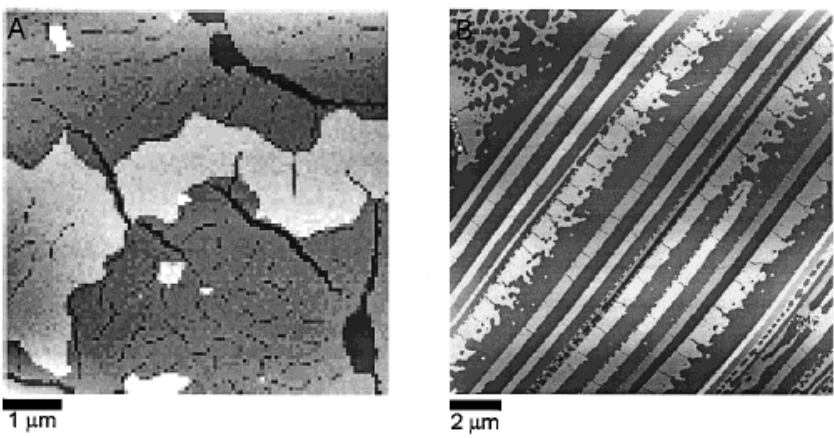

Figure 9. (a) AFM image of cracks in the monolayer/bilayer film of CdSe nanocrystals after drying. (b) AFM image of cracks in the thin film of CdSe nanocrystals after drying.

treatment, the spacing between cracks is described as:

$$
L \approx \frac{H}{q} \frac{s_{\mathrm{b}}}{s_{\mathrm{s}}}
$$

where $H$ is the height of the film, $s_{\mathrm{b}}$ is the breaking point of spring $1, s_{\mathrm{S}}$ measures the maximum distance that particles can be dragged before spring 2 breaks, and $q$ is the ratio between two spring constants.

In this treatment (and other spring models) $L / H$ measures the relative strength between two springs. The more easily interparticle bonds break, the more cracks will be generated. On the other hand, the more easily a particle can slide on the surface (lower resistance) the few cracks will occur. We have a $L / H$ ratio around 20:1, which indicates it is harder to break bonds between two nanocrystals than that between nanocrystals and graphite surface. This is consistent with our previous studies. ${ }^{11}$

Cracks also occur in a directional drying experiment. In Figure 9, the particles dried along step edges forming thin ribbons. The drying direction is perpendicular to the step edges. Parallel to the edges the stress is released. The graphite in the drying experiment was tilted to make this happen. Spacing between cracks is smaller in wider ribbons. We believe that this supports the idea of critical area in cracking. Stress density has to be larger than a critical value for cracks to appear.

\section{Conclusion}

$\mathrm{PbSe}$ nanocrystals in this study are three times larger than previous CdSe particles and have different coatings, yet the aggregate patterns formed at all coverages are the same as those formed by CdSe nanocrystals. A phase condensation model best describes these results. This suggests the use of nanocrystals on graphite as a prototype of a two-dimensional van der Waals system.
The particle-particle attractive energy (bonding strength) is estimated to about $3.5 k \mathrm{~T}$ in air, from the distribution histogram in the extremely dilute region. In the intermediate coverage, connectivity of particle domain and substrate domain show a sharp change across a narrow range. This enables the critical coverage of this system to be determined. At coverage larger than $64 \%$ cracking occurs due to the increased interparticle interaction in the dry form. Analysis of this cracking shows that the nanocrystal bonds to the graphite surface are weaker than the direct inter-nanocrystal bonds.

Acknowledgment. We thank Dr. C. Murray and Dr. W. Gaschler for PbSe nanocrystals. This work is supported by the Columbia MRSEC under Contract Number DMR-98-09687.

\section{References and Notes}

(1) For a recent review, see Collier, C. P.; Vossmeyer, T.; Heath, J. R. Annu. Rev. Phys. Chem. 1998, 49, 371.

(2) Ohara, P. C.; Leff, D. V.; Heath, J. R.; Gelbart, W. M. Phys. Rev. Lett. 1995, 75, 3466 .

(3) Korgel, B. A.; Fitzmaurice, D. Phys. Rev. Lett. 1998, 80, 3531.

(4) Korgel, B. A.; Fullam, S.; Connolly, S.; Fitzmaurize, D. J. Phys. Chem. B 1998, 102, 8479 .

(5) Mattoussi, H.; et al. Phys, Rev, B 1998, 58, 12.

(6) Rabani, E.; Egorov, S. A. J. Chem. Phys. 2001, 115 (8), 3437. 3323

(7) Leudtke, W. D.; Landman, U. J. Phys. Chem. 1996, 100 (32)

(8) Ohara, P. C.; Heath, J. R.; Gelbart, W. M. Angew. Chim., Int. Ed. 1997, 36 (10), 1078.

(9) Maillard, M.; Motte, L.; Ngo, A. T.; Pileni, M. P. J. Phys. Chem. B 2000, 104, 11871.

(10) Ge, G.; Brus, L. E. J. Phys. Chem. 2000, 104 (41), 9573.

(11) Ge, G.; Brus, L. E. Nanoletter 2001, 1 (4), 219.

(12) Israelachivili, J. N. Intermolecular and surface forces, 2nd ed. Academic: New York, 1992

(13) In this model, when closed shell configuration is formed, there is an extra $\alpha k T$ decrease in the free energy. In our size range of 3 to 7 , it can be neglected. Also, $\alpha$ should not be sensitive to experimentally determined $X_{1}$ because it is on the exponent position.

(14) Albanesi, E. A.; et al. Phys. Rev. B 2000, 61 (24), 16589

(15) Unpublished results.

(16) Connolly, S.; Fullam, S.; Korgel, B.; Fitzmaurice, D. J. Am. Chem. Soc. 1998, 120, 2969.

(17) Kim, B. S.; Avila, L.; Brus, L. E.; Herman, I. P. Appl. Phys. Lett. 2000, $76(25), 3715$.

(18) Sofonea, V.; Mecke, K. R. Eur. Phys. J. B 1999, 8, 99.

(19) Lin, X. M.; Jaeger, H. M.; Sorensen, C. M.; Klabunde, K. J. J. Phys. Chem. B 2001, 105, 3353.

(20) Voss, R. F.; Laibowitz, R. B.; Allessandrini, E. I. Phys. Rev. Lett. 1982, 49 (19), 1441.

(21) Kitsunezaki, S. Phys. Rev. 1999, E60 (6), 6449.

(22) Broughton, J.; Gilmer, G.; Weeks, J. Phys. Rev. 1982, B25, 46514669 .

(23) Toxvaerd, S. Phys. Rev. Lett. 1983, 51, 1971-1973

(24) Zollweg, J.; Chester, G. Phys. Rev. 1992, B46, 11186-11189.

(25) Alonso, J.; Fernandz, J. Phys. Rev. 1999, E59, 2059-2062.

(26) Lawn, B. Fracture of Brittle Solids; Cambridge University Press: New York, 1993.

(27) Skjeltorp, A. T.; Meakin, P. Nature 1988, 335 (29), 424

(28) Groisman, A.; Kaplan, E. Europhys. Lett. 1994, 25, 415.

(29) Barker, J. A.; Henderson, D.; Abrahm, F. F. Physica 1981, 106A, 226. 